\title{
A COMMUTANT OF AN UNBOUNDED OPERATOR ALGEBRA
}

\author{
ATSUSHI INOUE
}

\begin{abstract}
A commutant $\mathfrak{X}^{c}$ and bicommutant $\mathfrak{X}^{c c}$ of an unbounded operator algebra $\mathfrak{A}$ called a \#-algebra are defined. The first purpose of this paper is to investigate whether the bicommutant $\mathfrak{U}^{c c}$ of a \#-algebra $\mathfrak{A}$ is an $E W^{\#}$-algebra, as defined in [6], or not. The second purpose is to investigate the relation between $\mathfrak{A}^{c c}$ and topologies on a \#-algebra $\mathfrak{A}$.
\end{abstract}

In this paper let $\mathfrak{D}$ be a pre-Hilbert space with an inner product $(\mid)$ and $\mathfrak{h}$ the completion of $\mathfrak{D}$. Let $\mathfrak{L}(\mathfrak{D})$ denote the set of all linear operators on $\mathfrak{D}$ and $\mathfrak{L}^{\#}(\mathfrak{D})$ the set $\left\{A \in \mathfrak{L}(\mathfrak{D}) ; A^{*} \mathfrak{D} \subset \mathfrak{D}\right\}$. Every element $A$ of $\mathfrak{L}^{\#}(\mathfrak{D})$ is a closable operator on $\mathfrak{h}$ with the domain $\mathfrak{D}$. For each $A \in \mathfrak{L}^{\#}(\mathfrak{D})$, putting $A^{\#}=A^{*} / \mathfrak{D}$ (the restriction of $A^{*}$ onto $\mathfrak{D}$ ), the map $A \rightarrow A^{\#}$ is an involution on $\mathfrak{L}^{\#}(\mathfrak{D})$ and $\mathfrak{L}^{\#}(\mathfrak{D})$ is an algebra of operators on $\mathfrak{D}$ with the involution \#.

If $\mathfrak{A}$ is a \#-subalgebra of $\mathfrak{L}^{\#}(\mathfrak{D})$, then it is called a \#-algebra on $\mathfrak{D}$. In particular, $\mathfrak{L}^{\#}(\mathfrak{D})$ is called a maximal \#-algebra on $\mathfrak{D}$. We denote by $\bar{S}$ the smallest closed extension of $S \in \mathfrak{A}$ and by $\overline{\mathfrak{A}}$ the set $\{\bar{S} ; S \in \mathfrak{A}\}$. We set

$$
\mathfrak{A}_{b}=\{A \in \mathfrak{A} ; \bar{A} \in \mathfrak{B}(\mathfrak{h})\},
$$

where $\mathscr{B}(\mathfrak{h})$ denotes the set of all bounded linear operators on $\mathfrak{h}$, and call it the bounded part of $\mathfrak{A}$. A \#-algebra $\mathfrak{A}$ is called pure if $\mathfrak{A} \neq \mathfrak{A}_{b}$. A \#-algebra $\mathfrak{A}$ on $\mathfrak{D}$ is said to be symmetric if $\mathfrak{A}$ has an identity operator $I$ and $\left(I+A^{\#} A\right)^{-1} \in \mathfrak{A}_{b}$ for all $A \in \mathfrak{A}$. A symmetric \#-algebra $\mathfrak{U}$ on $\mathfrak{D}$ is called an $E W^{\#}$-algebra on $\mathfrak{D}$ over $\overline{\mathfrak{A}_{b}}$ if $\overline{\mathfrak{A}_{b}}$ is a von Neumann algebra.

A \#-algebra $\mathfrak{A}$ on $\mathfrak{D}$ is said to be closed (resp. selfadjoint) if

$$
\mathfrak{D}=\bigcap_{A \in \mathfrak{A}} \mathscr{D}(\bar{A}) \quad\left(\text { resp. } \mathscr{D}=\bigcap_{A \in \mathfrak{A}} \mathscr{D}\left(A^{*}\right)\right) .
$$

It is easy to show that if $\mathfrak{A}$ is a selfadjoint \#-algebra on $\mathfrak{D}$ then it is closed. By [6, Proposition 2.6], if $\mathfrak{A}$ is a closed symmetric \#-algebra, then it is selfadjoint.

In [6] we defined the commutant $\mathfrak{A}^{\prime}$ of a \#-algebra $\mathfrak{A}$ on $\mathfrak{D}$ as follows:

$$
\begin{aligned}
\mathfrak{U}^{\prime}=\{C \in \mathscr{B}(\mathfrak{h}) ;(C A \xi \mid \eta)= & \left(C \xi \mid A^{\#} \eta\right) \\
& \text { for all } A \in \mathfrak{A} \text { and } \xi, \eta \in \mathfrak{D}\} .
\end{aligned}
$$

From [6, Proposition 2.8] if $\mathfrak{A}$ is a selfadjoint \#-algebra on $\mathfrak{D}$ then $\mathfrak{A}^{\prime}$ is a von

Received by the editors December 13, 1976.

AMS (MOS) subject classifications (1970). Primary 46L20. 
Neumann algebra. Furthermore, for each $C \in \mathfrak{A}^{\prime}, A \in \mathfrak{A}$ and $\xi \in \mathfrak{D}$ we have $C \mathscr{D} \subset \mathfrak{D}$ and $C A \xi=A C \xi$. We define a new commutant $\mathfrak{U}^{c}$ and bicommutant $\mathfrak{A}^{c c}$ as follows:

$$
\begin{aligned}
\mathfrak{U}^{c} & =\left\{S \in \mathfrak{L}^{\#}(\mathfrak{D}) ; S A=A S \text { for all } A \in \mathfrak{A}\right\}, \\
\mathfrak{U}^{c c} & =\left\{A \in \mathcal{L}^{\#}(\mathfrak{D}) ; S A=A S \text { for all } S \in \mathfrak{U}^{c}\right\} .
\end{aligned}
$$

It is immediately shown that $\mathfrak{I}^{c}, \mathfrak{U}^{c c}$ are \#-algebras on $\mathfrak{D}$ and $\mathfrak{U}^{c c} \supset \mathfrak{A}$.

LEMMA 1. If $\mathfrak{A}$ is a selfadjoint \#-algebra on $\mathfrak{D}$, then:

(1) $\bar{T}$ is affiliated with $\left(\mathfrak{A}_{b}\right)^{\prime}\left(\right.$ written $\left.\bar{T} \eta\left(\mathfrak{A}_{b}\right)^{\prime}\right)$ for each $T \in \mathfrak{U}^{c}$ and $\left(\mathfrak{U}^{c}\right)_{b}=$ $\mathfrak{U}^{\prime} \subset\left(\underline{\mathfrak{U}}_{b}\right)^{\prime}$;

(2) $\bar{A} \eta \mathfrak{U}^{\prime \prime}$ for each $A \in \mathfrak{U}^{c c}$ and $\overline{\mathfrak{A}_{b}} \subset \overline{\left(\mathfrak{A}^{c c}\right)_{b}} \subset \mathfrak{A}^{\prime \prime}$.

LEMMA 2. If $\mathfrak{A}$ is a symmetric \#-algebra on $\mathfrak{D}$, then $\left(\mathfrak{A}_{b}\right)^{\prime}=\mathfrak{A}^{\prime}$ and $\left(\mathfrak{A}_{b}\right)^{\prime \prime}=\mathfrak{U}^{\prime \prime}$.

Proof. Clearly, $\mathfrak{U}^{\prime} \subset\left(\mathfrak{A}_{b}\right)^{\prime}$. Suppose that $C \in\left(\mathfrak{U}_{b}\right)^{\prime}$. Let $A \in \mathfrak{A}_{h}:=\{A \in$ $\left.\mathfrak{U} ; A^{\#}=A\right\}$. Since $\mathfrak{A}$ is symmetric, it is easily shown that $\left(I+A^{2}\right)^{-1}$, $A\left(I+A^{2}\right)^{-1} \in \mathfrak{A}_{b}$. For each $\xi, \eta \in \mathfrak{D}$ we have

$$
\left(C A\left(I+A^{2}\right)^{-1} \xi \mid \eta\right)=\left(A\left(I+A^{2}\right)^{-1} C \xi \mid \eta\right)=\left(A C\left(I+A^{2}\right)^{-1} \xi \mid \eta\right) .
$$

Since $\left(I+A^{2}\right)^{-1} \mathfrak{D}=\mathfrak{D}$, we get $C \in \mathfrak{U}^{\prime}$. Thus, $\left(\mathfrak{U}_{b}\right)^{\prime}=\mathfrak{U}^{\prime}$.

Proposition 1. Let $\mathfrak{A}$ be a closed symmetric \#-algebra on $\mathfrak{D}$. Then:

(1) $\mathfrak{A}^{c}$ is an $E W^{\#}$-algebra on $\mathfrak{D}$ over $\mathfrak{A}^{\prime}$;

(2) $\mathfrak{U}^{c c}=\left\{A \in \mathfrak{L}^{\#}(\mathfrak{D}) ; \bar{A} \eta \mathfrak{U}^{\prime \prime}=\left(\mathfrak{U}_{b}\right)^{\prime \prime}\right\}$;

(3) if $\left(\mathfrak{A}_{b}\right)^{\prime \prime} \mathfrak{D} \subset \mathfrak{D}$, then $\mathfrak{U}^{c c}$ is a closed $E W^{\#}$-algebra on $\mathfrak{D}$ over $\left(\mathfrak{U}_{b}\right)^{\prime \prime}$.

Proof. From [6, Proposition 2.6] $\mathfrak{A}$ is a selfadjoint \#-algebra on $\mathfrak{D}$. Hence this follows from Lemma 1.2.

Corollary. If $\mathfrak{A}$ is a closed $E W^{\#}$-algebra on $\mathfrak{D}$, then $\mathfrak{A}^{c}$ is an $E W^{\#}$. algebra on $\mathfrak{D}$ over $\mathfrak{U}^{\prime}$ and $\mathfrak{U}^{c c}$ is a closed $E W^{\#}$-algebra on $\mathfrak{D}$ over $\overline{\mathfrak{A}_{b}}$.

In this paper let $\mathscr{D}$ be an unbounded Hilbert algebra over $\mathscr{D}_{0}$ and $\mathfrak{h}\left(\mathscr{D}_{0}\right)$ the completion of $\mathscr{D}_{0}$. For the basic definitions and facts of unbounded Hilbert algebras the reader is referred to [7], [8]. Let $\mathscr{U}_{0}\left(\mathscr{D}_{0}\right)\left(\operatorname{resp} . \mathscr{V}_{0}\left(\mathscr{Q}_{0}\right)\right)$ be the left (resp. right) von Neumann algebra of the Hilbert algebra $\mathscr{D}_{0}$. Let $\pi_{0}$ (resp. $\pi_{0}^{\prime}$ ) be the left (resp. right) regular representation of $\mathscr{D}_{0}$ and $\phi_{0}$ (resp. $\phi_{0}^{\prime}$ ) the natural trace on $\mathscr{U}_{0}\left(\mathscr{D}_{0}\right)^{+}$(resp. $\left.\mathscr{V}_{0}\left(\mathscr{D}_{0}\right)^{+}\right)$. For each $x \in \mathfrak{h}\left(\mathscr{D}_{0}\right)$ we define $\pi_{0}(x)$ and $\pi_{0}^{\prime}(x)$ by

$$
\pi_{0}(x) \xi=\overline{\pi_{0}^{\prime}(\xi)} x, \quad \pi_{0}^{\prime}(x) \xi=\overline{\pi_{0}(\xi)} x \quad\left(\xi \in \mathscr{D}_{0}\right) .
$$

Then $\pi_{0}(x)$ and $\pi_{0}^{\prime}(x)$ are linear operators on $\mathfrak{h}\left(\mathscr{D}_{0}\right)$ with domain $\mathscr{D}_{0}$ and $\overline{\pi_{0}\left(x^{*}\right)}=\pi_{0}(x)^{*}, \overline{\pi_{0}^{\prime}\left(x^{*}\right)}=\pi_{0}^{\prime}(x)^{*}$. Putting $\left(\mathscr{D}_{0}\right)_{b}=\left\{x \in \mathfrak{h}\left(\mathscr{D}_{0}\right)\right.$; $\left.\overline{\pi_{0}(x)} \in \mathscr{B}\left(\mathfrak{h}\left(\mathscr{D}_{0}\right)\right)\right\},\left(\mathscr{D}_{0}\right)_{b}$ is a Hilbert algebra containing $\mathscr{D}_{0}$ and is called the maximal Hilbert algebra of $\mathscr{D}_{0}$ in $\mathfrak{h}\left(\mathscr{W}_{0}\right)$.

Let $\mathfrak{M}$ (resp. $\mathfrak{M}^{+}$) be the set of all measurable (resp. positive measurable) 
operators on $\mathfrak{h}\left(\mathscr{D}_{0}\right)$ with respect to $\mathscr{U}_{0}\left(\mathscr{D}_{0}\right)$. For each $T \in \mathfrak{M}^{+}$we set

$$
\begin{aligned}
\mu_{0}(T) & =\sup \left[\phi_{0}\left(\overline{\pi_{0}(\xi)}\right) ; 0 \leqslant \overline{\pi_{0}(\xi)} \leqslant T, \xi \in\left(\mathscr{D}_{0}\right)_{b}^{2}\right], \\
L^{p}\left(\phi_{0}\right) & =\left\{T \in \mathfrak{M} ;\|T\|_{p}:=\mu_{0}\left(|T|^{p}\right)^{1 / p}<\infty\right\}, \quad 1 \leqslant p<\infty, \\
L^{\infty}\left(\phi_{0}\right) & =\mathscr{U}_{0}\left(\mathscr{D}_{0}\right) .
\end{aligned}
$$

We define $L_{2}^{\omega}$-spaces with respect to $\phi_{0}$ and $\mathscr{D}_{0}$ as follows:

$$
L_{2}^{\omega}\left(\phi_{0}\right)=\bigcap_{2<p<\infty} L^{p}\left(\phi_{0}\right), \quad L_{2}^{\omega}\left(\mathscr{D}_{0}\right)=\left\{x \in \mathfrak{h} ; \overline{\pi_{0}(x)} \in L_{2}^{\omega}\left(\phi_{0}\right)\right\},
$$

respectively. By [7, Theorem 3.9] $L_{2}^{\omega}\left(\mathscr{D}_{0}\right)$ is maximal among unbounded Hilbert algebras containing $\mathscr{D}_{0}$ and is called the maximal unbounded Hilbert algebra of $\mathscr{D}_{0}$. Let $\pi_{2}^{\omega}$ (resp. $\left.\left(\pi^{\prime}\right)_{2}^{\omega}\right)$ be the left (resp. right) regular representation (i.e., $\left.\pi_{2}^{\omega}(x) y=x y,\left(\pi^{\prime}\right)_{2}^{\omega}(x) y=y x, x, y \in L_{2}^{\omega}\left(\mathscr{D}_{0}\right)\right)$ of $L_{2}^{\omega}\left(\mathscr{D}_{0}\right)$. We set

$$
\begin{aligned}
& \mathscr{U}_{0}\left(\mathscr{D}_{0}\right) / L_{2}^{\omega}\left(\mathscr{D}_{0}\right)=\left\{T / L_{2}^{\omega}\left(\mathscr{D}_{0}\right) ; T \in \mathscr{U}_{0}\left(\mathscr{D}_{0}\right)\right\}, \\
& \mathscr{V}_{0}\left(\mathscr{D}_{0}\right) / L_{2}^{\omega}\left(\mathscr{D}_{0}\right)=\left\{T^{\prime} / L_{2}^{\omega}\left(\mathscr{D}_{0}\right) ; T^{\prime} \in \mathscr{V}_{0}\left(\mathscr{D}_{0}\right)\right\} .
\end{aligned}
$$

Then $\pi_{2}^{\omega}(\mathscr{D}),\left(\pi^{\prime}\right)_{2}^{\omega}(\mathscr{D}), \mathscr{Q}_{0}\left(\mathscr{D}_{0}\right) / L_{2}^{\omega}\left(\mathscr{D}_{0}\right)$ and $\mathfrak{V}_{0}\left(\mathscr{D}_{0}\right) / L_{2}^{\omega}\left(\mathscr{D}_{0}\right)$ are \#-algebras on $L_{2}^{\omega}\left(\mathscr{D}_{0}\right)$. We denote by $\mathscr{Q}(\mathscr{D})$ (resp. $\left.\mathcal{T}(\mathscr{D})\right)$ a \#-algebra on $L_{2}^{\omega}\left(\mathscr{D}_{0}\right)$ generated by $\pi_{2}^{\omega}(\mathscr{D})$ and $\mathscr{U}_{0}\left(\mathscr{Q}_{0}\right) / L_{2}^{\omega}\left(\mathscr{D}_{0}\right)$ (resp. $\left(\pi^{\prime}\right)_{2}^{\omega}(\mathscr{D})$ and $\left.\widetilde{V}_{0}\left(\mathscr{D}_{0}\right) / L_{2}^{\omega}\left(\mathscr{D}_{0}\right)\right)$. Then $\mathscr{Q}(\mathscr{D})$ (resp. $\left.\mathscr{V}(\mathscr{D})\right)$ is an $E W^{\#}$-algebra on $L_{2}^{\omega}\left(\mathscr{D}_{0}\right)$ over $\mathscr{Q}_{0}\left(\mathscr{D}_{0}\right)$ (resp. $\left.\mathfrak{V}_{0}\left(\mathscr{D}_{0}\right)\right)$ and is called the left (resp. right) $E W^{\#}$-algebra of D.

THEOREM 1. Suppose that $\mathfrak{h}\left(\mathscr{D}_{0}\right)$ is not a Hilbert algebra, i.e., $\mathfrak{h}\left(\mathscr{D}_{0}\right) \neq\left(\mathscr{D}_{0}\right)_{b}$. Then:

(1) $\pi_{2}^{\omega}\left(\mathscr{D}_{0}\right)^{c}$ is a pure $E W^{\#}$-algebra on $L_{2}^{\omega}\left(\mathscr{D}_{0}\right)$ over $\mathscr{V}_{0}\left(\mathscr{D}_{0}\right)$ such that

$$
\pi_{2}^{\omega}\left(\mathscr{D}_{0}\right)^{c}=\left\{T \in \mathcal{L}^{\#}\left(L_{2}^{\omega}\left(\mathscr{D}_{0}\right)\right) ; \bar{T} \eta \mathscr{V}_{0}\left(\mathscr{D}_{0}\right)\right\} \supset \mathcal{V}\left(L_{2}^{\omega}\left(\mathscr{D}_{0}\right)\right) ;
$$

(2) $\pi_{2}^{\omega}\left(\mathscr{D}_{0}\right)^{c c}$ is a pure $E W^{\#}$-algebra on $L_{2}^{\omega}\left(\mathscr{D}_{0}\right)$ over $\mathscr{U}_{0}\left(\mathscr{D}_{0}\right)$ such that

$$
\pi_{2}^{\omega}\left(\mathscr{D}_{0}\right)^{c c}=\left\{A \in \mathfrak{L}^{\#}\left(L_{2}^{\omega}\left(\mathscr{D}_{0}\right)\right) ; \bar{A} \eta \mathscr{U}_{0}\left(\mathscr{D}_{0}\right)\right\} \supset \text { Q }\left(L_{2}^{\omega}\left(\mathscr{D}_{0}\right)\right) .
$$

Proof. (1) It is easily proved that $\pi_{2}^{\omega}\left(\mathscr{D}_{0}\right)^{c}=\left\{T \in \mathfrak{L}^{\#}\left(L_{2}^{\omega}\left(\mathscr{D}_{0}\right)\right)\right.$; $\left.\bar{T} \eta \mathscr{V}_{0}\left(\mathscr{D}_{0}\right)\right\}$. Since $\mathfrak{V}_{0}\left(\mathscr{D}_{0}\right) L_{2}^{\omega}\left(\mathscr{D}_{0}\right) \subset L_{2}^{\omega}\left(\mathscr{D}_{0}\right)$ and $\left(\pi^{\prime}\right)_{2}^{\omega}\left(L_{2}^{\omega}\left(\mathscr{D}_{0}\right)\right) L_{2}^{\omega}\left(\mathscr{D}_{0}\right) \subset$ $L_{2}^{\omega}\left(\mathscr{D}_{0}\right), \Upsilon_{0}\left(\mathscr{D}_{0}\right) / L_{2}^{\omega}\left(\mathscr{D}_{0}\right)$ and $\left(\pi^{\prime}\right)_{2}^{\omega}\left(L_{2}^{\omega}\left(\mathscr{D}_{0}\right)\right)$ are contained in $\pi_{2}^{\omega}\left(\mathscr{D}_{0}\right)^{c}$. Hence $\checkmark\left(L_{2}^{\omega}\left(\mathscr{D}_{0}\right)\right) \subset \pi_{2}^{\omega}\left(\mathscr{D}_{0}\right)^{c}$. Furthermore,

$$
\mathscr{V}_{0}\left(\mathscr{D}_{0}\right) \supset \overline{\left(\pi_{2}^{\omega}\left(\mathscr{D}_{0}\right)^{c}\right)_{b}} \supset \overline{\widetilde{V}\left(\mathscr{D}_{b}\right.}=\mathscr{V}_{0}\left(\mathscr{D}_{0}\right) \text {. }
$$

Thus $\pi_{2}^{\omega}\left(\mathscr{D}_{0}\right)^{c}$ is an $E W^{\#}$-algebra on $L_{2}^{\omega}\left(\mathscr{D}_{0}\right)$ over $\mathscr{V}_{0}\left(\mathscr{D}_{0}\right)$ containing $\checkmark\left(L_{2}^{\omega}\left(\mathscr{D}_{0}\right)\right)$. By [8, Theorem 3.4], $\mathscr{V}\left(L_{2}^{\omega}\left(\mathscr{D}_{0}\right)\right)$ is pure and it follows that $\pi_{2}^{\omega}\left(\mathscr{D}_{0}\right)^{c}$ is pure.

(2) This is proved in the same way as (1).

Corollary. If $\mathscr{D}$ is pure, then 


$$
\pi_{2}^{\omega}\left(\mathscr{D}_{0}\right)^{c}=\pi_{2}^{\omega}(\mathscr{D})^{c}=\pi_{2}^{\omega}\left(L_{2}^{\omega}\left(\mathscr{D}_{0}\right)\right)^{c}
$$

and

$$
\pi_{2}^{\omega}\left(\mathscr{D}_{0}\right)^{c c}=\pi_{2}^{\omega}(\mathscr{D})^{c c}=\pi_{2}^{\omega}\left(L_{2}^{\omega}\left(\mathscr{D}_{0}\right)\right)^{c c} .
$$

Furthermore, $\pi_{2}^{\omega}\left(\mathscr{D}_{0}\right)^{c}$ (resp. $\pi_{2}^{\omega}\left(\mathscr{D}_{0}\right)^{c c}$ ) is maximal among EW $W^{\#}$-algebras on $L_{2}^{\omega}\left(\mathscr{D}_{0}\right)$ over $\mathcal{T}_{0}\left(\mathscr{D}_{0}\right)\left(\right.$ resp. $\left.\mathscr{U}_{0}\left(\mathscr{D}_{0}\right)\right)$.

We call $\pi_{2}^{\omega}\left(\mathscr{D}_{0}\right)^{c c}$ (resp. $\left.\pi_{2}^{\omega}\left(\mathscr{D}_{0}\right)^{c}\right)$ the maximal left (resp. right) $E W^{\#}$. algebra of $\mathscr{D}_{0}$ and denote it by $\mathscr{T}^{l}\left(\mathscr{D}_{0}\right)\left(\right.$ resp. $\left.\mathscr{T}^{\gamma}\left(\mathscr{D}_{0}\right)\right)$.

Proposition 2. Let $\mathfrak{M}\left(\phi_{0}\right)$ (resp. $\mathfrak{M}\left(\phi_{0}^{\prime}\right)$ ) be the set of all $\phi_{0}$-measurable (resp. $\phi_{0}^{\prime}$-measurable) operators. Then:

$$
\begin{aligned}
& \mathscr{U}\left(L_{2}^{\omega}\left(\mathscr{D}_{0}\right)\right)=\left\{A \in \mathcal{L}^{\#}\left(L_{2}^{\omega}\left(\mathscr{D}_{0}\right)\right) ; \bar{A} \in \mathfrak{M}\left(\phi_{0}\right)\right\} ; \\
& \mathscr{V}\left(L_{2}^{\omega}\left(\mathscr{D}_{0}\right)\right)=\left\{T \in \mathcal{L}^{\#}\left(L_{2}^{\omega}\left(\mathscr{D}_{0}\right)\right) ; \bar{T} \in \mathfrak{M}\left(\phi_{0}^{\prime}\right)\right\} .
\end{aligned}
$$

Proof. (1) Suppose that $A \in \mathcal{L}^{\#}\left(L_{\underline{2}}^{\omega}\left(\mathscr{D}_{0}\right)\right)$ and $\bar{A} \in \mathfrak{M}\left(\phi_{0}\right)$. Let $\bar{A}=U|\bar{A}|$ be the polar decomposition of $\bar{A}$ and $|\bar{A}|=\int_{0}^{\infty} \lambda d E(\lambda)$ the spectral resolution of $|\bar{A}|$. Since $\bar{A}$ is $\phi_{0}$-measurable, $|\bar{A}|$ is $\phi_{0}$-measurable, and it follows that $E\left(\lambda_{0}\right)^{\perp}:=I-E\left(\lambda_{0}\right) \in \overline{\pi_{2}^{\omega}\left(\left(\mathscr{D}_{0}\right)_{b}\right)}$ for some $\lambda_{0}>0$, i.e., there exists an element $e_{\lambda_{0}}$ of $\left(\mathscr{D}_{0}\right)_{b}$ such that $E\left(\lambda_{0}\right)^{\perp}=\overline{\pi_{2}^{\omega}\left(e_{\lambda_{0}}\right)}$. Putting

$$
\begin{aligned}
& |A|=|\bar{A}| / L_{2}^{\omega}\left(\mathscr{D}_{0}\right), \\
& A_{0}=\left[\int_{0}^{\lambda_{0}} \lambda d E(\lambda)\right] / L_{2}^{\omega}\left(\mathscr{D}_{0}\right) \text { and } U_{0}=U / L_{2}^{\omega}\left(\mathscr{D}_{0}\right), \\
& |A| \in \mathcal{L}^{\#}\left(L_{2}^{\omega}\left(\mathscr{D}_{0}\right)\right) \text { and } A_{0}, U_{0} \in \mathscr{U}_{0}\left(\mathscr{D}_{0}\right) / L_{2}^{\omega}\left(\mathscr{D}_{0}\right) .
\end{aligned}
$$

Furthermore,

$$
A=U_{0}|A|=U_{0} A_{0}+\pi_{2}^{\omega}\left(U_{0}|A| e_{\lambda_{0}}\right) \in \mathscr{U}_{0}\left(\mathscr{D}_{0}\right) / L_{2}^{\omega}\left(\mathscr{D}_{0}\right)+\pi_{2}^{\omega}\left(L_{2}^{\omega}\left(\mathscr{D}_{0}\right)\right) .
$$

Hence, $A \in \mathcal{U}\left(L_{2}^{\omega}\left(\mathscr{D}_{0}\right)\right)$. Thus, $\left\{A \in \mathcal{L}^{\#}\left(L_{2}^{\omega}\left(\mathscr{D}_{0}\right)\right) ; \quad \bar{A} \in \mathfrak{M}\left(\phi_{0}\right)\right\} \subset$ $\mathcal{Q}\left(L_{2}^{\omega}\left(\mathscr{D}_{0}\right)\right)$. The reverse inclusion is obvious.

(2) This is proved in the same way as (1).

THEOREM 2. If $\mathscr{D}_{0}$ has an identity or $\mathfrak{h}\left(\mathscr{D}_{0}\right)$ is separable, then $\mathfrak{T}^{l}\left(\mathscr{D}_{0}\right)=$ $\mathscr{U}\left(L_{2}^{\omega}\left(\mathscr{D}_{0}\right)\right)$ and $\mathfrak{T C}^{\gamma}\left(\mathscr{D}_{0}\right)=\mathscr{V}\left(L_{2}^{\omega}\left(\mathscr{D}_{0}\right)\right)$.

Proof. If $\mathscr{D}_{0}$ has an identity, then this is easily proved. Suppose that $\mathfrak{h}\left(\mathscr{D}_{0}\right)$ is separable. P. G. Dixon [4, Theorem 5.3] has proved that each $E W^{*}$-algebra $\mathfrak{A}$ over a von Neumann algebra $\mathfrak{A}_{b}$ is contained in the algebra $\mathfrak{M}\left(\mathfrak{A}_{b}\right)$ of all measurable operators with respect to $\mathfrak{A}_{b}$. From Theorem $1, \overline{\mathfrak{R}^{l}\left(\mathscr{D}_{0}\right)}$ is an

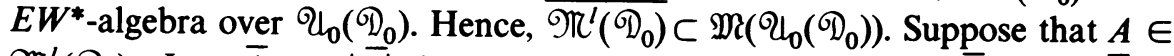
$\mathscr{T}^{l}\left(\mathscr{D}_{0}\right)$. Let $\bar{A}=U|\bar{A}|$ be the polar decomposition of $\bar{A}$ and $|\bar{A}|=$ $\int_{0}^{\infty} \lambda d E(\lambda)$ the spectral resolution of $|\bar{A}|$. Since $\bar{A}$ is measurable, $E_{0}:=E\left(\lambda_{0}\right)^{\perp}$ is a finite projection for some $\lambda_{0}>0$ and it follows that $\mathscr{U}_{0}\left(\mathscr{D}_{0}\right)_{E_{0}}$ is a finite von Neumann algebra. Furthermore, since $\mathfrak{h}\left(\mathscr{D}_{0}\right)$ is separable, $\mathscr{Q}_{0}\left(\mathscr{D}_{0}\right)_{E_{0}}$ is $\sigma$-finite. From [3, §6, Proposition 9], there exists a faithful normal finite trace $\chi_{0}$ on $\mathscr{U}_{0}\left(\mathscr{D}_{0}\right)_{E_{0}}^{+}$and it follows that there exists an isomorphism $\Psi$ of $\mathscr{U}_{0}\left(\mathscr{D}_{0}\right)_{E_{0}}$ onto a standard von Neumann algebra $\mathfrak{A}_{0}$ such that $\chi_{0}(T)=$ 
$\chi(\Psi(T))$ for every $T \in \mathscr{U}_{0}\left(\mathscr{D}_{0}\right)_{E_{0}}^{+}$, where $\chi$ denotes the natural trace on $\mathfrak{U}_{0}^{+}$ $[3, \S 6$, Theorem 2]. We set

$$
\left(\phi_{0}\right)_{E_{0}}(T)=\phi_{0}\left(T E_{0}\right), \quad T \in \mathscr{U}_{0}\left(\mathscr{D}_{0}\right)_{E_{0}}^{+} .
$$

Then it is easily proved that $\left(\phi_{0}\right)_{E_{0}}$ is a faithful normal semifinite trace on $\mathcal{U}_{0}\left(\mathscr{D}_{0}\right)_{E_{0}}^{+}$. Hence, from [3, $\S 6$, Theorem 2] there exists an isomorphism $\Phi$ of $\mathscr{Q}_{0}\left(\mathscr{D}_{0}\right)_{E_{0}}$ onto a standard von Neumann algebra $\mathfrak{B}_{0}$ such that $\left(\phi_{0}\right)_{E_{0}}(T)=$ $\phi(\Phi(T))$ for every $T \in \mathcal{U}_{0}\left(\mathscr{D}_{0}\right)_{E_{0}}^{+}$, where $\phi$ denotes the natural trace on $\mathfrak{B}_{0}^{+}$. Then the standard von Neumann algebras $\mathfrak{A}_{0}$ and $\mathfrak{B}_{0}$ are isomorphic. From $\left[3, \S 6\right.$, Theorem 4] $\mathfrak{A}_{0}$ and $\mathfrak{B}_{0}$ are spatially isomorphic. Since $\chi(T)<\infty$ for all $T \in \mathfrak{A}_{0}^{+}, \phi(T)<\infty$ for all $T \in \mathfrak{B}_{0}^{+}$. Hence, $\phi_{0}\left(E\left(\lambda_{0}\right)^{\perp}\right)=\phi(\Phi(I))<\infty$. Thus we can show that if $S$ is measurable with respect to $\mathscr{U}_{0}\left(\mathscr{D}_{0}\right)$ then $S$ is $\phi_{0}$-measurable. Hence, Theorem 2 follows from Proposition 2.

Next we shall investigate the relation between the commutants $\mathfrak{X}^{c}, \mathfrak{U}^{c c}$ of a \#-algebra $\mathfrak{A}$ on $\mathfrak{D}$ and topologies on $\mathfrak{A}$. The locally convex topology induced by seminorms: $P_{\xi, \eta}(T):=|(T \xi \mid \eta)|(\xi, \eta \in \mathscr{D})$ is called the weak topology on $\mathfrak{A}$. Let $\mathfrak{B}$ be a \#-algebra on $\mathfrak{D}$ containing $\mathfrak{A}$. We set

$$
\begin{aligned}
\mathscr{D}_{\infty}(\mathfrak{B})=\left\{\xi_{\infty}=\left(\xi_{1}, \xi_{2}, \ldots, \xi_{n}, \ldots\right) ; \xi_{n} \in \mathfrak{D}(n=1,2, \ldots)\right. \\
\left.\quad \text { and } \sum_{n=1}^{\infty}\left\|T \xi_{n}\right\|_{2}^{2}<\infty \text { for all } T \in \mathscr{B}\right\} .
\end{aligned}
$$

For each $\xi_{\infty}=\left(\xi_{1}, \xi_{2}, \ldots, \xi_{n}, \ldots\right)$ and $\eta_{\infty}=\left(\eta_{1}, \eta_{2}, \ldots, \eta_{n}, \ldots\right)$ in $\mathscr{D}_{\infty}(\mathfrak{B}), P_{\xi_{\infty}, \eta_{\infty}}(T):=\left|\sum_{n=1}^{\infty}\left(T \xi_{n} \mid \eta_{n}\right)\right|(T \in \mathfrak{A})$ is a seminorm on $\mathfrak{A}$. The locally convex topology on $\mathfrak{A}$ induced by the family $\left\{P_{\xi_{\infty}, \eta_{\infty}}() ; \xi_{\infty}, \eta_{\infty} \in \mathscr{D}_{\infty}(\mathfrak{B})\right\}$ of the seminorms is called the (BO)- $\sigma$-weak topology on $\mathfrak{A}$. In particular, the $\left(\mathfrak{L}^{\#}(\mathfrak{D})\right)$ - $\sigma$-weak topology on $\mathfrak{A}$ is simply called the $\sigma$-weak topology on $\mathfrak{A}$. It is easy to check that $\mathfrak{A}$ is a locally convex *-algebra under the involution \# and weak topology (or, $(\mathfrak{B})-\sigma$-weak topology). The strong topology is the locally convex topology induced by seminorms: $P_{\xi}(T)=\|T \xi\|(\xi \in \mathfrak{D})$. For each $\xi_{\infty}=\left(\xi_{1}, \xi_{2}, \ldots, \xi_{n}, \ldots\right) \in \mathscr{D}_{\infty}(\mathfrak{B}), P_{\xi_{\infty}}(T):=\left(\sum_{n=1}^{\infty}\left\|T \xi_{n}\right\|_{2}^{2}\right)^{1 / 2}(T \in$ $\mathfrak{U})$ is a seminorm on $\mathfrak{A}$. The locally convex topology induced by the

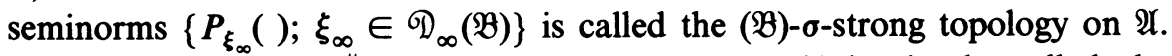
In particular, the $\left(\mathfrak{L}^{\#}(\mathfrak{D})\right)-\sigma$-strong topology on $\mathfrak{A}$ is simply called the $\sigma$-strong topology on $\mathfrak{A}$.

Proposition 3. If $\mathfrak{A}$ is a \#-algebra on $\mathfrak{D}$, then $\mathfrak{A}^{c}$ and $\mathfrak{A}^{c c}$ are closed in $\mathfrak{L}^{\#}(\mathfrak{D})$ under the weak topology.

COROLlary. $\mathfrak{T}^{l}\left(\mathscr{D}_{0}\right)$ and $\mathfrak{T}^{\gamma}\left(\mathscr{D}_{0}\right)$ are closed in $\mathfrak{L}^{\#}\left(L_{2}^{\omega}\left(\mathscr{D}_{0}\right)\right)$ under the weak topology.

THEOREM 3. If $\mathfrak{A}$ is a closed symmetric \#-algebra on $\mathfrak{D}$, then the following algebras, (1) $\sim(6)$, coincide with $\mathfrak{A}^{c c}$ :

(1) the weak closure $\left[\mathfrak{H}_{b}\right]^{\omega}$ of $\mathfrak{A}_{b}$ in $\mathfrak{L}^{\#}(\mathfrak{D})$;

(2) the strong closure $\left[\mathfrak{A}_{b}\right]^{5}$ of $\mathfrak{U}_{b}$ in $\mathfrak{L}^{\#}(\mathfrak{D})$; 
(3) the o-weak closure $\left[\mathfrak{A}_{b}\right]^{\sigma \omega}$ of $\mathfrak{A}_{b}$ in $\mathfrak{L}^{\#}(\mathfrak{D})$;

(4) the $\sigma$-strong closure $\left[\mathfrak{A}_{b}\right]^{\sigma s}$ of $\mathfrak{U}_{b}$ in $\mathcal{L}^{\#}(\mathfrak{D})$;

(5) the $\left(\mathfrak{A}^{c c}\right)$ - $\sigma$-weak closure $\left[\mathfrak{A}_{b}\right]^{\mathfrak{A}{ }^{c c}-\sigma \omega}$ of $\mathfrak{A}_{b}$ in $\mathfrak{L}^{\#}(\mathfrak{D})$;

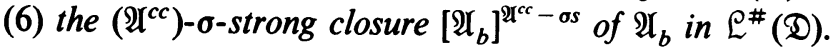

Proof. Clearly we have

$$
\begin{array}{cc}
{\left[\mathfrak{A}_{b}\right]^{s}} & \subset\left[\mathfrak{A}_{b}\right]^{\omega} \\
\cup & \cup \\
{\left[\mathfrak{U}_{b}\right]^{\sigma s}} & \subset\left[\mathfrak{U}_{b}\right]^{\sigma \omega} \\
\cup & \cup \\
{\left[\mathfrak{A}_{b}\right]^{\mathfrak{A}^{c c}-\sigma s}} & \subset\left[\mathfrak{U}_{b}\right]^{\mathfrak{A}^{c c}-\sigma \omega} .
\end{array}
$$

Hence we have only to show that $\mathfrak{A}^{c c} \subset\left[\mathfrak{A}_{b}\right]^{\mathfrak{A c c}^{c c}-\sigma s}$ and $\left[\mathfrak{A}_{b}\right]^{\omega} \subset \mathfrak{A}^{c c}$. For each $\xi_{\infty}=\left(\xi_{1}, \xi_{2}, \ldots\right) \in \mathscr{D}_{\infty}\left(\mathfrak{U}^{c c}\right)$ and $T \in \mathfrak{A}^{c c}$, putting $T_{\infty} \xi_{\infty}=\left(T \xi_{1}, T \xi_{2}, \ldots\right)$, $T_{\infty}$ is a linear operator on $\mathscr{D}_{\infty}\left(\mathfrak{U}^{c c}\right)$. It is easily shown that $\left(\mathfrak{U}^{c c}\right)_{\infty}:=\left\{T_{\infty} ; T\right.$ $\left.\in \mathfrak{A}^{c c}\right\}$ is a closed symmetric \#-algebra on $\mathscr{D}_{\infty}\left(\mathfrak{A}^{c c}\right)$ under the operations $S_{\infty}+T_{\infty}=(S+T)_{\infty}, \lambda T_{\infty}=(\lambda T)_{\infty}, S_{\infty} T_{\infty}=(S T)_{\infty}$ and $T_{\infty}^{\#}=\left(T^{\#}\right)_{\infty}$. Suppose $T \in \mathfrak{A}^{c c}$. Then $T_{\infty} \in\left(\mathfrak{A}^{c c}\right)_{\infty}$. From Proposition 1, $\bar{T}_{\infty}^{\infty} \eta\left(\left(\mathfrak{A}^{c c}\right)_{\infty}\right)_{b}^{\prime \prime}$. Hence, $T_{\infty} \in\left[\left(\left(\mathfrak{U}^{c c}\right)_{\infty}\right)_{b}^{\prime \prime}\right]^{3}$. It is easily proved that $\left(\left(\mathfrak{U}^{c c}\right)_{\infty}\right)_{b}^{\prime \prime}=\left(\mathfrak{A}_{b}^{\prime \prime}\right)_{\infty}$. Hence, $T_{\infty} \in\left[\left(\mathfrak{H}_{b}^{\prime \prime}\right)_{\infty}\right]^{s}$ and it follows that $T \in\left[\mathfrak{U}_{b}^{\prime \prime}\right]^{2 \mathfrak{A c}^{c c}-\boldsymbol{s}}$. Furthermore, $\mathfrak{A}_{b}$ is $\sigma-$ strongly dense in $\mathfrak{A}_{b}^{\prime \prime}$, and so $T \in\left[\mathfrak{A}_{b}\right]^{\mathfrak{A}^{c c}-o s}$. Thus, $\mathfrak{A}^{c c} \subset\left[\mathfrak{A}_{b}\right]^{\mathfrak{A l c}^{c c}-o s}$. From Proposition $3, \mathfrak{U}^{c c}$ is weakly closed and it follows that $\left[\mathfrak{U}_{b}\right]^{\omega} \subset \mathfrak{U}^{c c}$.

COROLlary. (1) $\mathfrak{T}^{l}\left(\mathscr{D}_{0}\right)$ equals the weak closure of $\pi_{2}^{\omega}\left(\mathscr{D}_{0}\right)$ in $\mathfrak{L}^{\#}\left(L_{2}^{\omega}\left(\mathscr{D}_{0}\right)\right)$.

(2) If $\mathscr{D}_{0}$ has an identity or $\mathfrak{h}\left(\mathscr{D}_{0}\right)$ is separable, then $\mathcal{Q}\left(L_{2}^{\omega}\left(\mathscr{D}_{0}\right)\right)$ equals the weak closure of $\pi_{2}^{\omega}\left(\mathscr{D}_{0}\right)$ in $\mathcal{L}^{\#}\left(L_{2}^{\omega}\left(\mathscr{D}_{0}\right)\right)$.

\section{REFERENCES}

1. R. Arens, The space $L^{\omega}$ and convex topological rings, Bull. Amer. Math. Soc. 52 (1946), 931-935.

2. R. M. Brooks, Some algebras of unbounded operators, Math. Nachr. 56 (1973), 47-62. 1969.

3. J. Dixmier, Les algèbres d'opérateurs dans l'espace hilbertien, 2é ed., Gauthier-Villars, Paris,

4. P. G. Dixon, Unbounded operator algebras, Proc. London Math. Soc. (3) 23 (1971), 53-69.

5. N. Dunford and J. T. Schwartz, Linear operators, Vol. II, Interscience, New York, 1963.

6. A. Inoue, On a class of unbounded operator algebras, Pacific J. Math. 65 (1976), 77-95.

7. __ On a class of unbounded operator algebras. II, Pacific J. Math. 66 (1976), 411-431.

8. __ On a class of unbounded operator algebras. III, Pacific J. Math. 69 (1977), 105-115.

9. Unbounded representations of symmetric *-algebras, J. Math. Soc. Japan 29 (1977), 219-232.

10. J. P. Jurzak, Simple facts about algebras of unbounded operators, J. Functional Analysis 21 (1976), 469-482.

11. G. Lassner, Topological algebras of operators, Rep. Mathematical Phys. 3 (1972), 279-293.

12. I. E. Segal, A noncommutative extension of abstract integration, Ann. of Math. (2) 57 (1953), 401-457.

Department of Applied Mathematics, Fukuoka University, Nanakuma, Fukuoka, Japan 